\title{
Looking beyond the WRIB Decennial Index of the White Papers in Bioanalysis
}

\author{
Wei Garofolo*,1 \& Natasha Savoie ${ }^{1}$ \\ ${ }^{1}$ CFABS, Montreal, QC, Canada \\ *Author for correspondence: Tel.: +1 514461 0877; weigarofolo@cfabs.org
}

\begin{abstract}
"The launch issue of Bioanalysis Journal opened with the 2009 White Paper and since then Bioanalysis has published all subsequent WRIB White Papers. These have been among the 'most read' articles in Bioanalysis for the past 10 years."
\end{abstract}

First draft submitted: 1 March 2019; Accepted for publication: 2 March 2019; Published online: 4 April 2019

Keywords: bioanalysis $\bullet$ biomarkers $\bullet$ discovery bioanalysis $\bullet$ emerging technologies $\bullet$ flow cytometry $\bullet$ gene therapy $\bullet$ hybrid LBA/LCMS • immunogenicity $\bullet$ LBA $\bullet$ LCMS • regulated bioanalysis $\bullet$ White Paper $\bullet$ WRIB

\section{Background: leading to the Decennial Index}

Starting as a one-day workshop attended by about 100 professionals focused on bioanalysis, WRIB (Workshop on Recent Issues in Bioanalysis) has since evolved into a 5-day full immersion in bioanalysis, biomarkers and immunogenicity attended by over 1000 professionals from pharmaceutical companies, CROs and regulatory agencies. The current format includes six full day-long advanced/specialized or training sessions (M1, M2, T1, Th1, F1 and F2) and three sequential main workshop days (Days 1, 2 and 3) with lectures by key international industry/regulator opinion leaders designed to facilitate sharing and discussing issues. WRIB now covers both smalland large-molecule bioanalysis, oligonucleotides and cell/gene therapy involving technologies such as LCMS, hybrid LBA/LCMS, LBA, cell-based assays/flow cytometry, qPCR and emerging technologies.

Each Workshop results in a White Paper divided in three parts, summarizing the recommendations and consensus items reached during the discussions, with contributions from over 50 subject matter experts [1-21]. The launch issue of Bioanalysis Journal opened with the 2009 White Paper [1] and since then Bioanalysis has published all subsequent WRIB White Papers. These have been among the 'most read' articles in Bioanalysis for the past 10 years [22].

\section{The Decennial Index}

In 2017, Bioanalysis published a Special Feature entitled "The Decennial Index of the White Papers in Bioanalysis: A Decade of Recommendations (2007-2016)" [23]. After 10 years of published White Papers, this article acts as a general index, organized alphabetically by topic, to easily consult all the recommendations and their evolution over time.

Natasha Savoie, co-author of the Index and WRIB Team Member summarized the ' 5 Ws' of the Decennial Index, below [24].

The ' 5 Ws' of the Decennial Index

- 1. What is the Decennial Index of the White Papers in Bioanalysis?

- It is an alphabetical list of topics consolidating the evolution of 10 years of global WRIB bioanalytical community discussions and recommendations.

\section{- 2. Why was the Index created?}

- WRIB gets a lot of questions from bioanalytical professionals from all over the globe via the website every year on various topics that they are interested in, and a lot of them have already been answered with recommendations. WRIB wanted to put a paper together so that the global bioanalytical community has something to refer to in order to get their answers.

- 3. Who will this Index appeal to most? 
- This index has been designed from answering the questions of the global bioanalytical community. Hence, bioanalytical scientists and anybody who works in discovery or regulated bioanalysis, immunogenicity and/or biomarkers who are interested in the scientific and regulatory evolution of these discussions or in established and emerging technologies involved in bioanalysis will find this index very useful.

\section{- 4. Where can the Index be accessed?}

- You just have to go to the Bioanalysis ournal home page [25] to browse it directly online with a searchable index or you can download a free and secure PDF document containing all the links to get to the different topics. You can also simply type/google 'Bioanalysis' in any web browsers to be able to access the Decennial Index searchable index directly and quickly from laptops and smartphones.

\section{- 5. When do you anticipate updating the index?}

- We do not think we are going to do it every year. We are looking at every 3-5 years right now but it will depend how quickly innovation in bioanalysis is being embraced and science and regulations evolve as well as how the discussions and recommendations progress every year.

\section{WRIB and... beyond}

Over the next decade, WRIB will continue its well-established tradition of providing novel solutions in drug discovery, drug development and promoting innovation in method development by covering a wide range of hot topics in bioanalysis, biomarkers and immunogenicity suggested directly by attendees from pharmaceutical/biopharmaceutical and biotechnology companies, contract research organizations and regulatory agencies worldwide [26] based on:

\section{"Designing the next WRIB together: Built by you for you."}

WRIB will keep its unique format as a meeting of Key Opinion Leaders with the primary goal of serving the global bioanalytical community by authoring the White Paper in Bioanalysis each year. Authors will include industry experts and regulatory representatives, with input from open panel discussions among the presenters, regulators and attendees in order to reach consensus.

Hence, WRIB will continue its mission to address and distill hot topics into a series of relevant recommendations, as in the previous decade, by gathering a diverse group of international industry and regulatory authority experts working on both small and large molecules to facilitate sharing and discussions focused on:

\section{"Improving quality, increasing regulatory compliance and achieving scientific excellence on bioanalytical} issues."

The active participation of regulatory agency representatives will continue to be a fundamental part of the WRIB:

\section{"Where Regulators \& Industry Convene"}

WRIB will still include three sequential main workshop days, and several additional advanced/specialized or training full-day sessions that together with the main workshop days span an entire week in order to allow exhaustive and thorough coverage of all major issues in bioanalysis, biomarkers and immunogenicity.

See you at the WRIB, and beyond...

Wei, Natasha and the rest of the WRIB Team

Financial \& competing interests disclosure

The authors have no relevant affiliations or financial involvement with any organization or entity with a financial interest in or financial conflict with the subject matter or materials discussed in the manuscript. This includes employment, consultancies, honoraria, stock ownership or options, expert testimony, grants or patents received or pending, or royalties.

No writing assistance was utilized in the production of this manuscript.

\section{References}

1. Savoie N, Booth BP, Bradley T et al. The 2nd Calibration and Validation Group Workshop on recent issues in Good Laboratory Practice bioanalysis. Bioanalysis 1(1), 19-30 (2009).

2. Savoie N, Garofolo F, van Amsterdam P et al. 2009 White Paper on recent issues in regulated bioanalysis from the 3rd Calibration and Validation Group Workshop. Bioanalysis 2(1), 53-68 (2010). 
3. Savoie N, Garofolo F, van Amsterdam P et al. 2010 White Paper on recent issues in regulated bioanalysis and global harmonization of bioanalytical guidance. Bioanalysis 2(12), 1945-1960 (2010).

4. Garofolo F, Rocci M, Dumont I et al. 2011 White Paper on recent issues in bioanalysis and regulatory findings from audits and inspections. Bioanalysis 3(18), 2081-2096 (2011).

5. DeSilva B, Garofolo F, Rocci M et al. 2012 White Paper on recent issues in bioanalysis and alignment of multiple guidelines. Bioanalysis 4(18), 2213-2226 (2012).

6. Stevenson L, Rocci M, Garofolo F et al. 2013 White Paper on recent issues in bioanalysis: "hybrid" - the best of LBA \& LC/MS. Bioanalysis 5(23), 2903-2918 (2013).

7. Fluhler E, Hayes R, Garofolo F et al. 2014 White Paper on recent issues in bioanalysis: a full immersion in bioanalysis (Part 1 - small molecules by LCMS). Bioanalysis 6(22), 3039-3049 (2014).

8. Dufield D, Neubert H, Garofolo F et al. 2014 White Paper on recent issues in bioanalysis: a full immersion in bioanalysis (Part 2 hybrid LBA/LCMS, ELN \& regulatory agencies' input). Bioanalysis 6(23), 3237-3249 (2014).

9. Stevenson L, Amaravadi L, Myler H et al. 2014 White Paper on recent issues in bioanalysis: a full immersion in bioanalysis (Part 3 - LBA and immunogenicity). Bioanalysis 6(24), 3355-3368 (2014).

10. Welink J, Fluhler E, Hughes N et al. 2015 White Paper on recent issues in bioanalysis: focus on new technologies and biomarkers (Part 1 - small molecules by LCMS). Bioanalysis 7(22), 2913-2925 (2015).

11. Ackermann B, Neubert H, Hughes N et al. 2015 White Paper on recent issues in bioanalysis: focus on new technologies and biomarkers (Part 2 - hybrid LBA/LCMS and input from regulatory agencies). Bioanalysis 7(23), 3019-3034 (2015).

12. Amaravadi L, Song A, Myler H et al. 2015 White Paper on recent issues in bioanalysis: focus on new technologies and biomarkers (Part 3 - LBA, biomarkers and immunogenicity). Bioanalysis 7(24), 3107-3124 (2015).

13. Yang E, Welink J, Cape S et al. 2016 White Paper on recent issues in bioanalysis: focus on biomarker assay validation (BAV) (Part 1 small molecules, peptides and small molecule biomarkers by LCMS). Bioanalysis 8(22), 2363-2378 (2016).

14. Song A, Lee A, Garofolo F et al. 2016 White Paper on recent issues in bioanalysis: focus on biomarker assay validation (BAV): (Part 2 hybrid LBA/LCMS and input from regulatory agencies). Bioanalysis 8(23), 2457-2474 (2016).

15. Richards S, Amaravadi L, Pillutla R et al. 2016 White Paper on recent issues in bioanalysis: focus on biomarker assay validation (BAV): (Part 3 - LBA, biomarkers and immunogenicity). Bioanalysis 8(23), 2475-2496 (2016).

16. Welink J, Yang E, Hughes N et al. 2017 White Paper on recent issues in bioanalysis: aren't BMV guidance/guidelines 'scientific'? (Part 1 - LCMS: small molecules, peptides and small molecule biomarkers). Bioanalysis 9(22), 1807-1825 (2017).

17. Neubert H, Song A, Lee A et al. 2017 White Paper on recent issues in bioanalysis: rise of hybrid LBA/LCMS immunogenicity assays (Part 2: hybrid LBA/LCMS biotherapeutics, biomarkers \& immunogenicity assays and regulatory agencies' inputs). Bioanalysis 9(23), 1895-1912 (2017).

18. Gupta S, Richards S, Amaravadi L et al. 2017 White Paper on recent issues in bioanalysis: a global perspective on immunogenicity guidelines \& biomarker assay performance (Part 3 - LBA: immunogenicity, biomarkers and PK assays). Bioanalysis 9(24), 1967-1996 (2017).

19. Welink J, Xu Y, Yang E et al. 2018 White Paper on Recent Issues in Bioanalysis: 'a global bioanalytical community perspective on last decade of incurred samples reanalysis (ISR)' (Part 1 - small molecule regulated bioanalysis, small molecule biomarkers, peptides \& oligonucleotide bioanalysis). Bioanalysis 10(22), 1781-1801 (2018).

20. Neubert H, Olah T, Lee A et al. 2018 White Paper on Recent Issues in Bioanalysis: focus on immunogenicity assays by hybrid LBA/LCMS and regulatory feedback (Part 2 - PK, PD \& ADA assays by hybrid LBA/LCMS \& regulatory agencies' inputs on bioanalysis, biomarkers and immunogenicity). Bioanalysis 10(23), 1897-1917 (2018).

21. Stevenson L, Richards S, Pillutla R et al. 2018 White Paper on Recent Issues in Bioanalysis: focus on flow cytometry, gene therapy, cut points and key clarifications on BAV (Part 3 - LBA/cell-based assays: immunogenicity, biomarkers and PK assays). Bioanalysis 10(24), 1973-2001 (2018).

22. Future Science. Most read Bioanalysis articles. www.future-science.com/journals/bio/mostread

23. Garofolo W, Savoie N. The Decennial Index of the White Papers in Bioanalysis: 'a decade of recommendations (2007-2016)'. Bioanalysis 9(21), 1681-1704 (2017).

24. Bioanalysis Zone: The decennial index of White Papers in Bioanalysis: an interview with Natasha Savoie. www.bioanalysis-zone.com/2018/06/22/decennial-index-white-papers-bioanalysis-interview-natasha-savoie/

25. Bioanalysis. www.future-science.com/journal/bio

26. WRIB: Submit Your Questions to Regulators. www.wrib.org/questions-to-regulators.php 
